\title{
Rapid Cutaneous Metastases from a Rectal Primary: A Case Report
}

\author{
Rebecca Stoner ${ }^{1}$, Joanne Cunningham ${ }^{2}$ and Adnan Qureshi ${ }^{3 *}$ \\ ${ }^{1}$ Department of Colorectal Surgery, Milton Keynes University Hospital NHS Trust, UK \\ ${ }^{2}$ Department of Pathology, Milton Keynes University Hospital NHS Trust, UK \\ ${ }^{3}$ Department of Colorectal Surgery, Milton Keynes University Hospital NHS Trust, UK \\ *Corresponding author: Adnan Qureshi, Department of Pathology and Colorectal Surgery, Milton Keynes University Hospital NHS Trust, UK
}

Submission: April 11, 2018; Published: April 30, 2018

\begin{abstract}
The development of cutaneous metastases from gastrointestinal malignancies are rare, but they have been previously reported secondary to primary colorectal adenocarcinoma in relation to advanced disease. This case report describes a phenomenon of rapidly developing cutaneous and liver metastases within weeks, from a stage 3 rectal adeno carcinoma operated with curative intent.
\end{abstract}

A literature review of similar cases was made and contrasted to the rapidity and stage of this aggressive tumour. The current guidelines are also reflected on, as well as the rate of recurrence with different modalities of treatment.

Keywords: Rectal carcinoma; Cutaneous metastases; Gastrointestinal malignancies; Adenocarcinoma; Liver metastases

\section{Introduction}

This case report discusses the rapid metastases of a rectal carcinoma to the skin and liver soon after curative resection of primary tumour. Cutaneous metastases from gastrointestinal malignancy are rare, and has been observed in $4-6 \%$ of colorectal adenocarcinoma cases $[1,2]$. When these cancers do metastasise to the skin, they mostly present as firm, painless and discrete nodules $[2]$.

There are several mechanisms of cutaneous metastasis in theory, ranging from direct implantation to lymphatic or haematogenous spread $[3,4]$. There are at least 25 case reports in English literature on cutaneous colorectal metastasis to date.

This case is rare because it was stage 3 colorectal adenocarcinoma with curative resection, and developed cutaneous metastasis within 5 weeks of original surgery, leaving one to suspect that metastasis can present that early and quickly even after curative surgery.

Further studies and tumour type behaviour is needed in future to determine these high risk colorectal adenocarcinoma with potential of early widespread metastases.

\section{Case Report}

The patient was a 47 year old lady with severe learning difficulties due to cerebral palsy with a performance status of four, due to contractures of the upper and lower limbs, rendering her immobile. She was admitted under the emergency surgery team with a single episode of rectal bleeding and a palpable rectal mass. She underwent examination under anaesthesia and flexible sigmoidoscopy. Histology from biopsies confirmed a moderately differentiated adeno carcinoma of the rectum.

A staging CT and MRI scan revealed a tumour in the left lateral position involving the mid and lower rectum extending into muscularis propria. No extra luminal disease or lymphadenopathy was identified. Final radiological staging was Duke's stage B, T2N0M0. She underwent surgery with curative intent during the same admission.

The patient had an APER due to technical reasons caused by her limb contractures. Post-operative histology showed a tumour size of $47 \mathrm{~mm}$, 3 out of the 20 lymph nodes sampled were found to be positive. One lymph node was found very close to the circumference margin anteriorly at $0.1 \mathrm{~mm}$, thus making it a $\mathrm{R} 1$ resection. The 
longitudinal margins were clear. Histology showed perineural invasion but no evidence of vascular invasion. Final staging was moderately differentiated adenocarcinoma rectum with focal areas of poor differentiation, Dukes C1, T2N1M0 (Figure 1A). It was decided that the patient was not for further adjuvant treatment due to performance status.

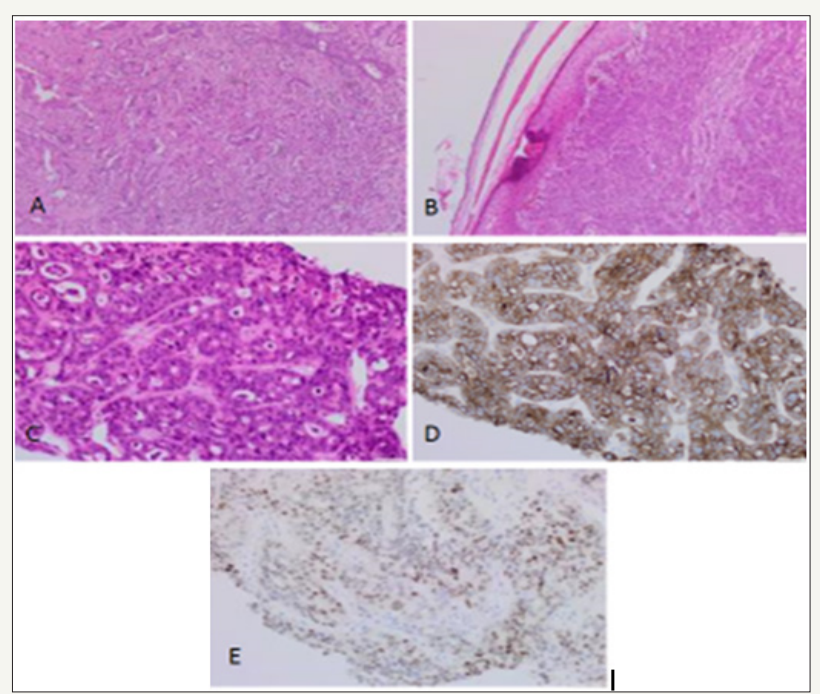

Figure 1: A: Moderately differentiated adenocarcinoma of the rectum. B: Skin showing a solid dermal nodule comprising poorly differentiated adenocarcinoma cells. C: Liver which is infiltrated by poorly differentiated adenocarcinoma. D: CK20 immuno histochemistry on the liver shows strong cytoplasmic expression. E: CDX2 immuno histochemistry on the liver shows patchy strong nuclear expression.

The patient had a smooth recovery and was awaiting social input. However, five weeks post operatively she was found clinically to be septic with a raised white blood cell count and C-reactive protein. She was treated empirically with antibiotics for suspected sepsis.

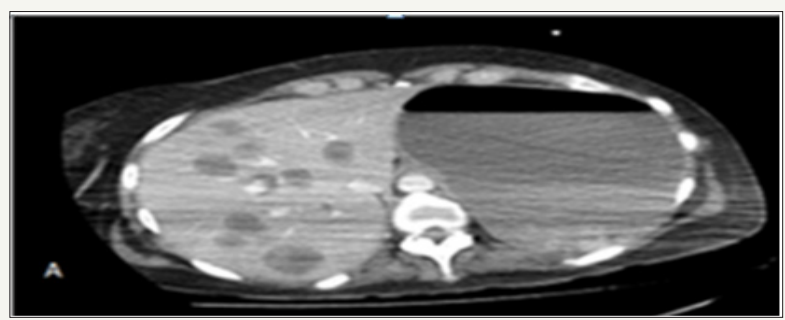

Figure 2A: Multiple Liver Metastases.

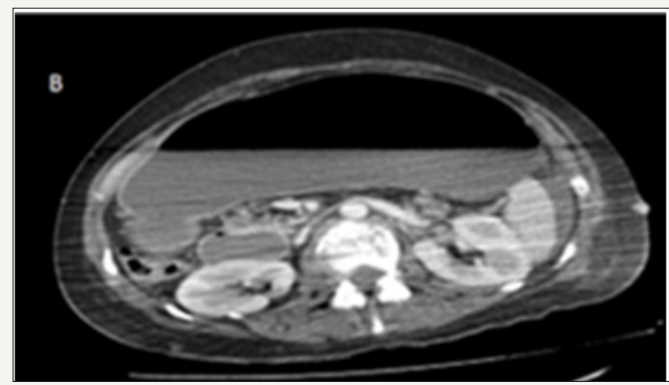

Figure 2B: Two cutaneous Metastatic nodules.
Clinically, she developed two enlarged lymph nodes in the posterior triangle of the neck, left supra clavicular fossa and left axilla. ACT scan was performed to look for source of sepsis the scan showed, enhancing nodules in the subcutaneous fat and in the gluteal muscles with multiple hypoechoic liver lesions and peritoneal deposits, suspected to be metastatic disease (Figure 2).

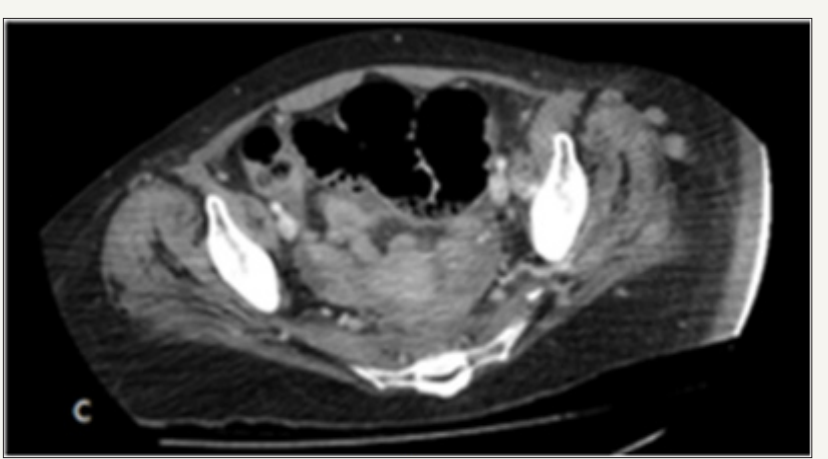

Figure 2C: Deep subcutaneous metastatic deposits.

Biopsy of a liver and skin lesion were performed. Both histology samples were reported as being poorly differentiated adenocarcinoma (Figure 1B \& 1C). Immuno histochemistry showed the tumour cells expressed CK20 and CDX2 (Figure 1D \& 1E). They were negative for CK7. These were in keeping with metastatic poorly differentiated adenocarcinoma from a colorectal primary.

The patient was seen by the palliative team after discussion with her family and was moved to a hospice where she passed away due to metastatic disease within 8 weeks after the surgery for moderately differentiated adenocarcinoma stage 3 , where prognosis is normally in years.

\section{Discussion}

A study by Abrahms, et al. [5] observing the metastases at autopsy in 1000 patients found that the commonest site of metastasis for rectal carcinoma is the liver $47 \%$ followed by the lung at $38 \%$. Only $3 \%$ were found to have skin metastasis [1,5]. Brown and Clark quotes $58.8 \%$ of their patients with rectal cancer have no metastases, though they reflected on the fact that a significant number of their patients did not survive long past resection or colostomy formations [6].

Risk of extra-nodal metastases is up to $20 \%$ for stage I and II colorectal cancers within 5 years of surgery [7]. Isolated tumour cells in regional lymph nodes have been implicated [8], and it is recommended that at least 12 to 15 lymph nodes be sampled for regional staging [9].

There have been other cases of rectal adenocarcinoma with cutaneous metastasis in the literature. Our case is unique as the patient developed cutaneous metastasis within five weeks of curative surgery. A similar case reported in the literature which describes a 55 year old gentleman with T3N2M0 rectal adenocarcinoma presenting 18 months following surgery and chemotherapy, with subcutaneous metastatic nodules [10]. A similar phenomenon 
described by Wang et al. [11] in a 76 year old lady with T4aN1Mx poorly differentiated adenocarcinoma with lymph node metastasis, also developed subcutaneous nodules on the patient's back [11]. This occurred within one month of surgery, in this case report the patient already had locally advanced T4 disease with unmapped metastasis, necessitating neo adjuvant chemotherapy which was not administered due to impending bowel obstruction. Another case reporting a 44 year old lady who presented with a number of well defined, painless subcutaneous nodules which were found to be metastases, from a primary T4 N2 M1, sigmoid adenocarcinoma [12].

Current guidelines do not recommend adjuvant chemotherapy for stage 2 disease [13]. A retrospective study observing patients with $\mathrm{T} 1$ and $\mathrm{T} 2$ disease treated by local excision alone found that $37 \%$ of patients with $\mathrm{T} 2$ disease, had a recurrence at 54 months post op, with the average time being 18 months [14,15].

This case is unique in that metastases to both skin and liver occurred within 5 weeks of curative surgery for a stage 3 predominantly moderately differentiated rectal adenocarcinoma which developed rapidly following surgery. The exact mechanism to which cutaneous metastases from rectal carcinoma occurs is uncertain, and further research is needed to determine the risk factors for this.

\section{References}

1. Lookingbill DP, Spangler N, Helm KF (1993) Cutaneous metastases in patients with metastatic carcinoma: A retrospective study of 4020 patients. J Am Acad Dermatol 29(2): 228-236.

2. Guanziroli E, Coggi A, Venegoni L, Fanoni D, Ercoli G, et al. (2017) Cutaneous metastases of internal malignancies: An experience from a single institution. Eur J Dermatol 27(6): 609-614.

3. Royston D, David G Jackson (2009) Mechanisms of lymphatic metastasis in human colorectal adenocarcinoma. The Journal of Pathology 217(5): 608-619.
4. Weiss L, Grundmann E, Torhorst J, Hartveit F, Moberg I, et al. (1986) Haematogenous metastastic patterns in colonic carcinoma: An analysis of 1541 necropsies. J Pathol 150(3): 195-203.

5. Abrams HL, Spiro R, Goldstein N (1950) Metastases in carcinoma. Analysis of 1000 autopsied cases. Cancer 3(1): 74-85.

6. Brown CE, Warren S (1938) Visceral metastasis from rectal carcinoma. Surg Gynecol Obstet 66: 611-621.

7. Bilchik A, Nissan A, Wainberg Z, Shen P, McCarter M, et al. (2010) Surgical quality and nodal ultrastaging is associated with long-term diseasefree survival in early colorectal cancer: An analysis of 2 international multicenter prospective trials. Ann Surg 252(3): 467-474; discussion 474-476.

8. Mescoli C, Albertoni L, Pucciarelli S, Giacomelli L, RussoVM, et al. (2012) Isolated tumor cells in regional lymph nodes as relapse predictors in stage I and II colorectal cancer. J Clin Oncol 30(9): 965-971.

9. Compton CC, Fielding LP, Burgart LJ, Conley B, Cooper HS, et al. (2000) Prognostic factors in colorectal cancer.College of american pathologists consensus statement 1999. Arch Pathol Lab Med 124(7): 979-994.

10. de Miguel Valencia MJ, Fraile Gonzalez M, Yague Hernando A, Oteiza Martinez F, Ciga Lozano MA (2013) Cutaneous metastases of rectal cancer. Anales Del Sistema Sanitario De Navarra 36(3): 557-561.

11. Wang DY, Feng Ye, Jian Jiang Lin, Xiao Xu (2017) Cutaneous metastasis: A rare phenomenon of colorectal cancer. Ann Surg Treat Res 93(5): 277280.

12. Llaguna OH, Desai P, Fender AB, Zedek DC, Meyers MO, et al. (2010) Subcutaneous metastatic adenocarcinoma: An unusual presentation of colon cancer - case report and literature review. Case Rep Oncol 3(3): 386-390.

13. Benson AB $3^{\text {rd }}$, Schrag D, Somerfield MR, Cohen AM, Figueredo AT, et al. (2004) American society of clinical oncology recommendations on adjuvant chemotherapy for stage II colon cancer. J Clin Oncol 22(16): 3408-3419.

14. Garcia Aguilar J, Mellgren A, Sirivongs P, Buie D, Madoff RD, et al. (2000) Local excision of rectal cancer without adjuvant therapy: a word of caution. Ann Surg 231(3): 345-351.

15. Gmitter TL, Dhawan SS, Phillips MG, Wiszniak J (1990) Cutaneous metastases of colonic adenocarcinoma. Cutis 46(1): 66-68.
Creative Commons Attribution 4.0 International License

For possible submissions Click Here

\section{Submit Article}

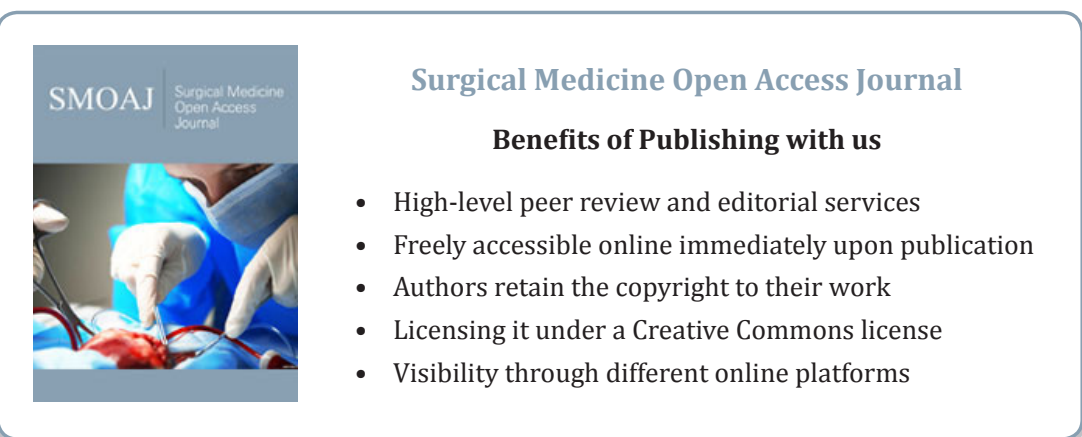

